\title{
Development of an Indexing Media Filtration System for Long Duration Space Missions
}

\author{
Juan H. Agui* \\ NASA Glenn Research Center, Cleveland, $\mathrm{OH}, 44135$ \\ and \\ R. Vijayakumar ${ }^{\dagger}$ \\ Aerfil, Liverpool, NY, 13088
}

\begin{abstract}
The effective maintenance of air quality aboard spacecraft cabins will be vital to future human exploration missions. A key component will be the air cleaning filtration system which will need to remove a broad size range of particles derived from multiple biological and material sources. In addition, during surface missions any extraterrestrial planetary dust, including dust generated by near-by ISRU equipment, which is tracked into the habitat will also need to be managed by the filtration system inside the pressurized habitat compartments. An indexing media filter system is being developed to meet the demand for long-duration missions that will result in dramatic increases in filter service life and loading capacity, and will require minimal crew involvement. The filtration system consists of three stages: an inertial impactor stage, an indexing media stage, and a high-efficiency filter stage, packaged in a stacked modular cartridge configuration. Each stage will target a specific range of particle sizes that optimize the filtration and regeneration performance of the system. An $1 / 8^{\text {th }}$ scale and full-scale prototype of the filter system have been fabricated and have been tested in the laboratory and reduced gravity environments that simulate conditions on spacecrafts, landers and habitats. Results from recent laboratory and reducegravity flight tests data will be presented. The features of the new filter system may also benefit other closed systems, such as submarines, and remote location terrestrial installations where servicing and replacement of filter units is not practical.
\end{abstract}

\section{Nomenclature}

$A \quad=$ filter element cross-sectional area

$C_{c} \quad=$ slip coefficient

$d_{50}=$ particle diameter at $50 \%$ efficiency

$\mathrm{d}_{\mathrm{j}} \quad=$ impactor jet diameter

$K_{p} \quad=$ medium permeability

$L \quad=$ length of rectangular jet impactor

$\mathrm{P} \quad=$ penetration

$\mathrm{p}=$ pressure

$Q \quad=$ flow rate

Stk $k_{50}=$ Stokes number at $d_{50}$

$U \quad=$ jet velocity

$W \quad=$ width of rectangular jet impactor

$w \quad=$ width of impactor plate, or collection strip

$\varepsilon=$ Particle collection efficiency

$\mu \quad=$ viscosity

* Aerospace Engineer, Fluid Physics and Transport Branch, 21000 Brookpark Rd, Mail Stop 77-5, Cleveland, OH, AIAA Member .

${ }^{\dagger}$ President 
$\begin{array}{lll}\rho_{p} & =\text { particle density } \\ \tau & = & \text { relaxation time }\end{array}$

\section{Introduction}

$\mathrm{I}_{\mathrm{e}}^{\mathrm{n}}$ order to provide safe and sustainable, breathable air aboard crewed space vehicles and extraterrestrial outposts,

ffective dust mitigation techniques are needed for remote and long duration space operation. Filtration systems are a vital component of life support systems. They serve to remove nuisance and harmful particulates and ultimately provide clean air and comfort for human health. However, filtering of airborne particles under the environmental constraints and conditions of spacecraft and planetary surface system poses unique challenges. The filter system not only must be capable of removing common particulate matter such as skin flakes, hair and clothing fibers, and particulate matter from food and hygiene operations, but it must also remove particulate debris from operating machinery and equipment, and planetary dust tracked in by extravehicular activity (EVA). Thus the system must be capable of filtering particle sizes spread over several orders of magnitude. Furthermore, since servicing or replacing filters in space is not a trivial task, the system is also required to last extraordinary life times, of the order of several years.

Filtration technologies are well established in terrestrial applications. However, they rely on replacement units for continued operation. On long duration crewed space missions this practice may not be very feasible. Specifically, high efficiency fibrous filters, which make up the bulk of filter elements on air handling systems, are excellent at providing greater than $99.9 \%$ particle capturing efficiency, but are virtually impossible to regenerate without damage to the filter medium. This is because dust particles adhere tenaciously to the fibers throughout the depth of the filter. However, HEPA (High Efficiency Particulate Air) filters for example if properly pre-filtered can have extensive service lives. Therefore it is proposed that regeneration needs to be accommodated through alternate filtration or separation means in conjunction with high efficiency filtration. This paper describes a novel multi-stage filter system that has been designed under an Innovative Partnership Program (IPP) project between NASA and Aerfil. Prototypes of the Indexing Media Filtration system (also known as the scroll filter) have been developed. The filter system is designed to meet the demand for long-duration missions by providing dramatic increases in filter service life and loading capacity, and will require minimal crew involvement. The filtration system consists of three stages: an inertial impactor stage, an indexing media stage, and a high-efficiency filter stage, packaged in a stacked modular cartridge configuration. Each stage targets a specific range of particle sizes that optimize the filtration and regeneration performance of the system. This modular design also provides the flexibility to add more stages of filters for performance optimization, and to meet design and operational requirements of any space or sealed environment mission.

The objective of this investigation was to characterize the overall performance of the new filtration system. The filter system was prototyped and then tested for its filtration performance and self-cleaning operation. Details of the design and features of the filter system are presented. The "Results and Discussion" section provides the first assessment of the filter system.

\section{Hardware Description}

\section{A. The filter system}

Two different scale prototypes of the scroll filter system were designed and fabricated, and subsequently tested under laboratory and low-g conditions. First, a reduced-scaled (approximately $1 / 8^{\text {th }}$ scale, based on the ratio of cross-sectional areas) prototype, $10.8 \mathrm{~cm} \times 10.8 \mathrm{~cm}$ in cross-section, of the filtration system was fabricated through stereolithography (SLA)-based rapid-prototyping. The prototype filtration system consisted of three stages: an inertial impactor stage, an indexing media stage, and a high-efficiency filter stage, packaged in a stacked modular cartridge configuration. Subsequently, a full scale prototype of the filter system was fabricated through an international partner (contracted through Aerfil). A picture of the full scale prototype is provided in Fig. 1a, while a another picture comparing the size of the full and scaled prototypes is shown in Fig. 1b. The full-scale prototype has a cross-sectional area of $30 \mathrm{~cm}$ x $30 \mathrm{~cm}$ and was built structurally from aluminum sheet metal. It has the same staged design as the scaled prototype. The main components shown in Fig. 1a include the test articles, the impactor and scroll stages, and the entrance and conic duct sections used for testing purposes. The high-efficiency filter component, described above, was not included because it was not used in testing. Both prototypes were designed to be flexible to accommodate different performance characteristic filter elements. They were designed to operate at a nominal flow rate in atmospheric air of $0.047 \mathrm{~m}^{3} / \mathrm{s}\left(100 \mathrm{cfm}\right.$, full scale performance) and $0.0057 \mathrm{~m}^{3} / \mathrm{s}(12 \mathrm{cfm})$ 
respectively for the full scale and $1 / 8^{\text {th }}$ scale prototypes. The associated face velocity approaching the filter surface in both cases is $0.5 \mathrm{~m} / \mathrm{s}$, which is a the nominal face velocity used with high efficiency filters for terrestrial use.

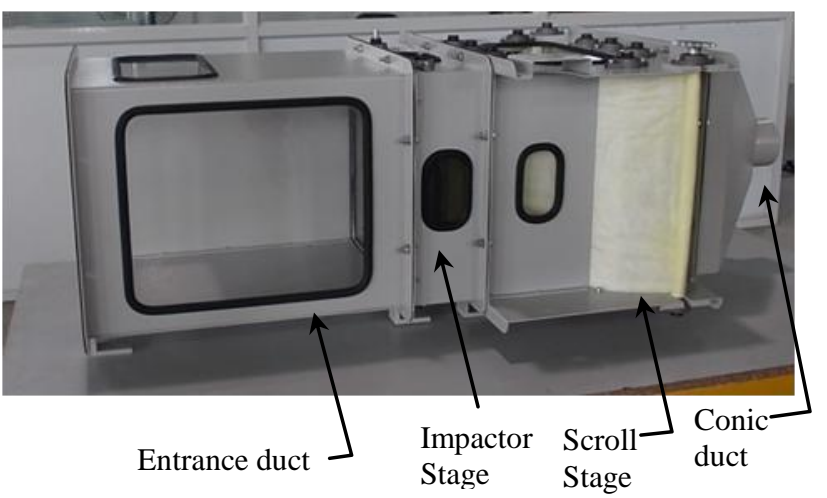

(a)

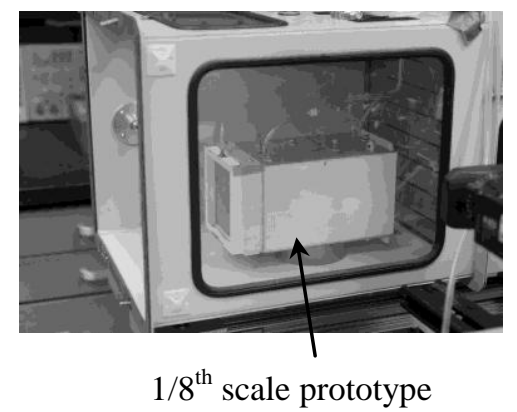

(b)

Figure 1: Prototype hardware (a) full scale Indexing Media Filter System, (b) full and reduced-scale prototypes (scale model placed inside full scale model for size comparison).

Each stage of multi-stage air filtration system plays a specific role in the filtration process. The inertial filter stage was designed to capture the largest particulates in order to reduce the loading on the indexing media filter and, depending on configuration, the HEPA filter stage, thereby prolonging the filter system's service life. The indexing media filter, or scroll stage, captures intermediate particle sizes (typically a few microns). Since this filter stage is expected to become heavily loaded with particulate matter over long operations, due to high loading events, it must be regenerated or replenished by dust mitigation means involving very low-maintenance components. To minimize maintenance, the indexing media filter will be replenished by means of a motorized spool that rolls up the dust laden portion of the filter medium on one side of the filter housing, thereby removing the accumulated dust and replenishing the dust-laden filter section with fresh media. As an added feature, in addition to containing and storing the collected dust material within the filter medium as it is tightly wound up in a roll, the rolled medium can be subsequent analyzed to determine dust generation (or loading) rates and dust composition throughout the mission. The HEPA filter is the last stage of filtration and therefore its role is to capture the remaining (smallest) particulates with very high efficiency. The HEPA stage was not installed for testing since HEPA air filter performance is well characterized throughout the industry.

The principle of the impaction stage is based on the transport behavior of particles impinging on bluff flat surfaces normal to the flow. A conceptual schematic of the flow with particle trajectories is shown in Fig. 2. The flow is accelerated through the aperture on the orifice plate and then is suddenly redirected near the flat surface of the impaction plate, or collection strip, directly behind the aperture. The high turning angle causes relatively large particles to impact the plate while the smaller particles, which are well entrained in the flow, pass around the plate surface and continue downstream with the flow. Equation 1 provides a relationship between the $d_{50}$ cut size (i.e. the particle diameter at which 50\% of particles of this diameter are collected on the impaction plate and the other 50\% penetrate through) and the particle transport (Stoke's number) and flow (flow rate) parameters. ${ }^{1}$

$$
d_{50}=\sqrt{\frac{9 \eta D_{j}^{2}\left(S t k_{50}\right)}{\rho_{p} Q}}
$$

Or for a rectangular jet,

\footnotetext{
* The maximum attainable flow velocity of the full-scale prototype was $0.4 \mathrm{~m} / \mathrm{s}$ with the size of blower used and the impactor and scroll filter stages installed.
} 


$$
d_{50}=\sqrt{\frac{9 \eta W^{2} L\left(S t k_{50}\right)}{\rho_{p} Q}}
$$

Where $d_{j}$ is the jet diameter for a round jet, $W$ and $L$ are the width and length of the orifice plate aperture in the case of a rectangular jet, $C_{c}$ is the Cunningham Slip Correction factor, $\rho_{p}$ is the particle density, $Q$ is the flow rate and $S t k_{50}$ is the Stokes number evaluated at the $d_{50}$ particle diameter. The Stokes number is defined as,

$$
S t k=\frac{\tau U}{\left(D_{j} / 2\right)}
$$

The variable $\tau$ is the relaxation time which is the time needed by the particle to adjust, or relax, to changes in flow conditions, defined as,

$$
\tau=\frac{\rho_{p} d^{2} C_{c}}{18 \mu}
$$

and $\mu$ is the fluid viscosity.

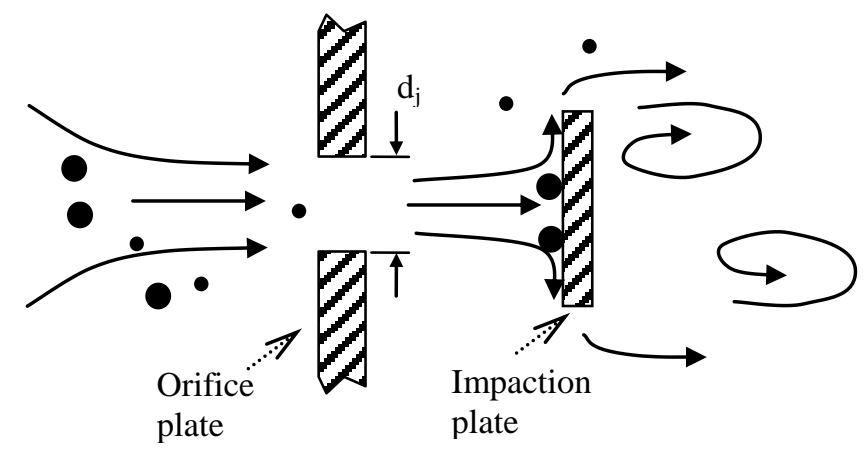

Figure 2: Flow schematic through an inertial impactor

Based on these equations, the jet diameter, or width and length for a rectangular jet, is chosen based on the $d_{50}$ particle filtration requirement and flow conditions. The classical plot of the collection efficiency of an impactor, represented in Fig. 3a, is characterized by a steep rise at the $d_{50}$ particle size from zero efficiency to $100 \%$ efficiency for particle sizes larger than $d_{50}$. The performance of the impactor is not very sensitive to the width of the collection surface or the separation distance between the orifice and collection surface. The hardware or system constraints and requirements can dictate these dimensions, while the width of the collectin surface needs to be at least slightly wider than $d_{j}$. In the scale prototype, the orifice openings consisted 4 sets of approximately two hundred $1 \mathrm{~mm}$ diameter straight hole perforations in a long rectangular raster pattern that were micro-machined on a thin $1.5 \mathrm{~mm}$ thick orifice plate. Alternately, because of the challenge of scaling up this type of fabrication and to minimize air resistance the orifice openings on the full scale prototype consisted of eight $3 \mathrm{~mm}$ wide slots machined on the orifice plate. A nominal $d_{50}$ of $5 \mu \mathrm{m}$ was selected for the current filter designs. 
The impactor stage incorporates endless bands or belts that span across most of the width of the duct just behind (downstream) of the orifice plate. A thin layer of grease on the surface of the belt facing the incoming flow minimizes particle rebound and facilitates their cleaning. The belts ride on pulleys mounted on vertical spindles placed internally near the inside of the duct side walls. The user has access to the tops of the spindles that extend above the top wall and can manually rotate the spindles. Alternatively, the spindles can be motorized and electronically controlled for remote or autonomous operation. As one of the spindles is rotated from above, the belts are translated across the width of the duct and eventually cycle all the way around the belt loop. In the full scale prototype the belts encounter a wiper or scrapper that removes the layer of particles and grease as they cycle through the loop. Although there is a clear utility in using the wiper, its presence in the internal duct volume may introduce flow complexities that cannot easily be discerned.

The scroll filter stage provides excess filter media that is supplied and collected on spools. A servo motor is activated to start scrolling the medium while exposing a fresh surface of the medium to the flow. The filter medium is threaded through a series of internal spindles that allow the medium to form pleats inside the duct. Pleats are very beneficial in air filtration because they increase the filter surface area and reduce the media velocity, both aspects leading to better filter performance. All internal spindles and outer spools are mounted on roller bearings to facilitate the spooling operation and reduce the motor power requirements. The design of the system allows for installation of any grade of filter media to meet the desired filtration specification of the scroll filter. In the prototypes tested, commercial grade filter media with performance in the MERV (Minimum efficiency reporting value) 11 to 15 range, typically used to capture submicron to micron size particulates, were installed on the scroll filter stage. Nominally MERV 10 to 11 filters provide collections efficiencies of 60 to $70 \%$ in the $1 \mu \mathrm{m}$ to $3 \mu \mathrm{m}$ particle range, while MERV $12-14$ filters achieve capturing efficiencies, in the $0.3 \mu \mathrm{m}$ to $1 \mu \mathrm{m}$ range, of $80 \%$ to $90 \%$. In the upper range, MERV 14-15 filters offer efficiencies of $90 \%$ to $95 \%$. Fibrous filter media are used ubiquitously in air filtration because it is cost-effective and also provides an effective method of filtration known as depth filtration. Fig. 3b shows a typical filtration performance curve for depth filters. Depth filters can capture most particles sizes with virtually $100 \%$ efficiency, while a portion of the particles in the narrow band between 0.03 and $0.5 \mu \mathrm{m}$, typically, can penetrate through. This is due to a less than complementary transition from the diffusional mode of filtration (at the smallest particles sizes) to the inertial mode of filtration (at the largest particle sizes), that permits a small amount of particle penetration in this size range. These effects are represented in Fig. 3b. However, despite the success and practicality of depths filters in terrestrial applications, depths filters are highly susceptible to particle saturation and even caking which drives up their air resistance and in turn requires more system power to continue in operation until the filter is changed. The scroll filter is in effect a self-changing or self-cleaning filter system where the filter medium is autonomously (or through user control) replaced.

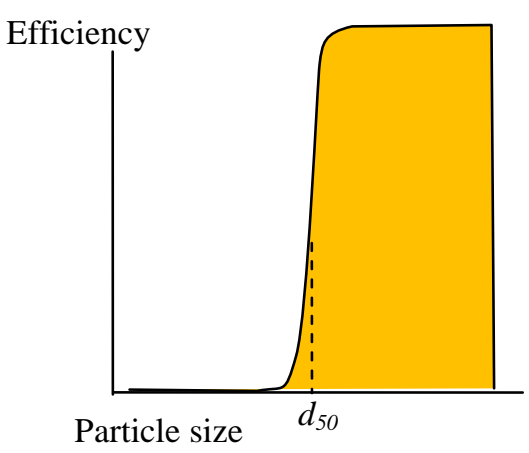

(a)

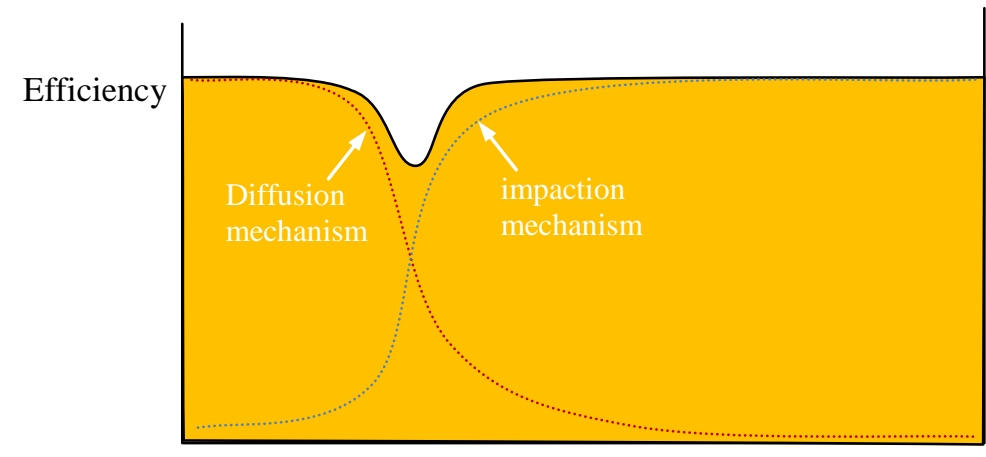

Particle size

(b)

Figure 3: Typical capturing efficiency curve for: (a) an impactor; (b) a depth filter showing the contribution to efficiency from the diffusional and inertial impaction capturing mechanisms.

\section{B. Testing platform}

The prototypes were assembled and tested on a portable test rig which was used in the laboratory setting as well as installed on the Zero-g aircraft for low gravity testing. A picture of the test rig configured for flight appears in Fig. 4. The experimental rig was designed as a multi-use test stand to assess the performance of filter systems and 
components. The scaled prototype was tested first and was internally mounted to an existing duct assembly from a previous flight experiment. Therefore, the flow had to internally transition between the larger duct dimensions to the that of the scaled prototype. The flow was driven by a bank of four axial fans in a square configuration at the end of the duct length. Alternatively, the full scale prototype was mounted on the same test rig but no flow transition section was required in the entrance region because the ducting of the filter system itself was used to interface directly to the flow source. The flow was driven by a high capacity commercial HEPA vacuum cleaner that suctioned the flow through the entrance duct and filter stages. The vacuum cleaner inlet interfaced with the ducted sections through a transition conic section shown in Fig. 1a. In both prototypes a HEPA filter element was installed at the start of inlet duct to remove most of the room- or cabin-air particulates that would have interfered with the challenge aerosol stream produced by the particle generator. The bottom shelf of the test rig provides the power and signal avionics required for testing. Measurements of pressure drops across filter elements, entrance duct flow speeds, particle counts and sizes, and imaging of the particle flow were conducted on the rig. Pressure measurements were performed with low pressure differential pressure transducers. Flow velocities were measured in clean air, before particles were introduced in the flow, using hot-wire based velocity probes. A pair of optical particle counters (OPC) were used to simultaneously measure the particle counts upstream and downstream of the filter elements. These OPC's permit simultaneous measurement of six channels of particle sizes from 0.3 to 10 microns. Sampling times were limited to 20 seconds in low-g testing, and ranged from 20 seconds to 2 minutes in the laboratory tests. The pressure and velocity data were logged through a USB-interfaced data logger. Samples were taken at $1 \mathrm{kHz}$ sampling rate for 20 seconds to 2 minutes to average out any transients. One high definition (HD) camcorder along with a high intensity Light Emitting Diode (LED) light source, for illumination, and some optical components were used to image the particle flow in the upstream region as well as image the indexing/scrolling operations.

A custom designed particle generator initially designed for close-system operation was used in all tests. A description of the particle generator is provided in Ref. 2. It provides sustained solid particle injections without introducing additional air flow into the system. Since it produces a wake flow, instead of a jet flow as with some commercial particle injectors, it can produce faster spreading and mixing of the particles as they advect downstream. While the particle generator performed well in both sets of tests, it was found by subsequent analysis that the shorter distance between the particle generator and the first (upstream) sampling probe in the scale prototype case most likely did not allow for sufficient particle dispersion at this measuring station. As a consequence the upstream particle counts were considered to be significantly skewed to larger values. This is issue is discussed later in the "Results and Discussion" section.

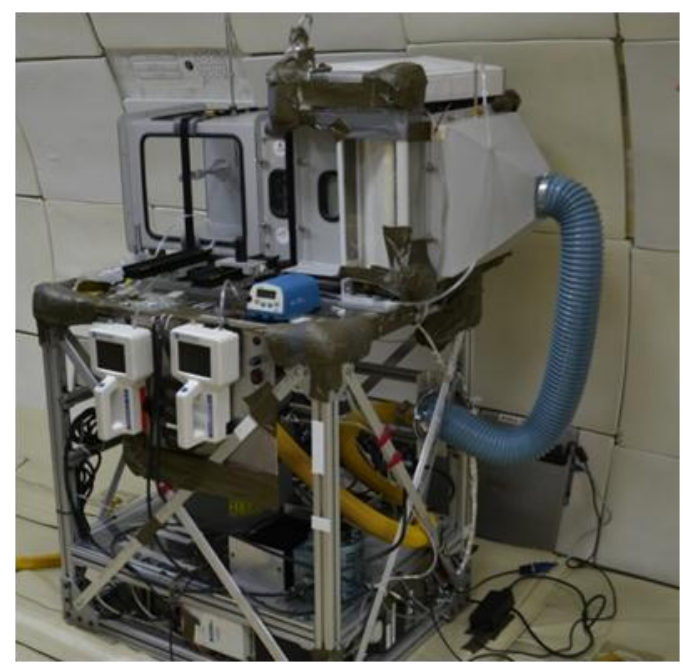

Figure 4: Test rig with full scale prototype installed in the Zero-G aircraft. 


\section{Materials and Methods}

Methods for testing conventional filters, such as the filter media used in the current set of tests, are fairly standard. In the industry, these methods rely on well established, often large, testing facilities that provide uniform air and particulate flows. Similar methods will be applied to the new filter system, noting however that under the current test plan testing was confined to a smaller footprint testing platform and that it involved the use of certain non-conventional, non-media, components. Also to achieve relevant sealed environment conditions, alternate approaches to particle injection or generation methods were also taken.

The two main performance parameters that describe filter functionality is permeability and collection efficiency. High permeability coupled with high particle capturing efficiency is a desired characteristic of high efficiency filtration. These parameters are typically determined by subjecting the filter media to well controlled flow conditions and challenging it with aerosol standards (see ASHRAE ${ }^{3}$, IEST ${ }^{4}$, ISO $29463^{5}$ test standards). In these test protocols the pressure drop across the filter element, and particle counts in the flow upstream and downstream of the filter element are used in the calculation of the performance parameters. However, since our interest is in the overall performance of the filter system under relevant sealed environment conditions, certain variations of the standard test protocol were adapted. First, the filter system was tested in the customized test rig described in the previous section which constrained some of the testing techniques. An additional level of flow characterization was required of the impaction collection device which was expected to significantly alter the flow in the surrounding flow field. The hydrodynamic performance of the impactor stage was characterized by its performance (or resistance) curves, pressure vs. flow rate. This characterization is relevant because it relates to the level of power usage to drive the flow. To account for the scroll filter's pleated configuration, permeability for the scroll filter medium is given in terms of the flow rate per unit area at a specified pressure drop, i.e. $K=Q / A$ at a prescribed pressure drop, $\Delta p$. Lastly, because of the interest in testing under relevant environmental conditions and over a range of particle sizes, a lunar dust simulant was used instead of using particle standards.

Particle penetration, $P$, is determined from the ratio of particle counts upstream and downstream of the filter element, i.e.

$$
P=\frac{N_{\text {downstream }}}{N_{\text {upstream }}}
$$

Where $\mathrm{N}$ is the particle count or total number of particles sampled. The associated collection efficiency is then given by,

$$
\varepsilon=1-P
$$

High collection efficiency indicates that the downstream counts are low compared to the upstream counts (low penetration), while low collection efficiency is obtained when the downstream counts are similar in level to the upstream counts (high penetration).The challenge aerosols used in this case, were derived from JSC-1af lunar dust simulant and JSC-Mars-1 equivalent simulants. JSC-1af is a basaltic based mineral powder with $20 \mathrm{wt} \%$ of the particles below $10 \mu \mathrm{m}$ in size. ${ }^{6}$ The Martian simulant is an equivalent JSC-Mars 1 simulant derived from palagonite mineral from Mauna Kea, Hawaii. These simulants have an average density of $2.9 \mathrm{~g} / \mathrm{cm}^{3}$ which tends to make them settle relatively quickly during testing. To mitigate this, the entrance region where the particles are introduced is made shorter than standard entrance or development regions for filter testing, that typically require an entrance length of several characteristic duct lengths (i.e. duct diameter or width). The shorter entrance length however can reduce the mixing and spreading of the aerosols needed for proper testing prior to the measuring stations. The other mitigating approach is to test in low or micro-gravity conditions which can reduce or eliminate particle settling. Although this helps with minimizing settling, the dispersion of the particles prior to reaching the first filter stage is still constrained by the shorter entrance length. In fact the length of the entrance duct was kept short in anticipation of low-g testing, in which often there is a benefit in containing the overall size of the flight payload for manifesting purposes.

Limited low-g testing of both prototypes were performed on the Zero-G Corporation aircraft through NASA's Flight Opportunities Program. The aircraft performs parabolic arc maneuvers that transition between low-g levels (zero to planetary surface gravity levels) to high-g (approximately 1.8 earth g's) levels. Multiple simulated low-g gravity periods are performed during a typical flight, which provides the researchers with about 20 seconds each period of steady low-g levels for testing their hardware. 
Table 1 provides a list of test configurations presented in the results section.

Table 1: Filter test configuration

\begin{tabular}{|l|l|l|l|l|}
\hline Configuration & Prototype & Impactor & Scroll filter & Environment \\
\hline 1 & $1 / 8^{\text {th }}$ scale & Impactor & No media & Lab. and flight \\
\hline 2 & $1 / 8^{\text {th }}$ scale & Scroll media & MERV 13 and 15 & Lab. and flight \\
\hline 3 & $1 / 8^{\text {th }}$ scale & Impactor and Scroll filter & MERV 13 and 15 & Lab. and flight \\
\hline 4 & Full scale & Impactor and Scroll filter & No media, just scroll housing & Lab. and flight \\
\hline 5 & Full scale & Impactor and Scroll filter & MERV 11 & Lab. and flight \\
\hline
\end{tabular}

\section{Results and Discussion}

The laboratory and flight test data are presented in this section. Since the data is preliminary in nature, discussions and interpretations of the results are provided. The two subsections that follow focus on the flow and particle transport performance and filtration performance of the prototype filter systems.

\section{A. Flow and Particle Transport Performance.}

The flow performance of the impactor stage was one of the first performance characterisitcs investigated and the results are given in the graphs in Fig. 5. The graphs show the pressure drop produced across the impactor stages at various velocities under laboratory ambient conditions. There is clear distinction between the scaled and full scale prototype in the magnitude of pressure drop. There seems to be significant differences in the slopes of the two curves, with distinctly steeper slopes attributed to the scaled prototype (note that due to the large difference in nominal flow rates of the two prototypes their respective curves could not be plotted on the same basis scale). This, most likely, was due to the design of the orifice plate on the scale prototype which consists of several hundred $1 \mathrm{~mm}$ diameter size holes in front of each band, as compared to the larger slots on the full scale prototype. As is seen in both cases, although more so with the scaled prototype, the pressure drop rises rather significantly with small changes in face velocities. At about $0.52 \mathrm{~m} / \mathrm{s}$ the pressure drop has reached $125 \mathrm{~Pa}$ or 0.5 inches of water on the full scale prototype, and $150 \mathrm{~Pa}$ for the scaled prototype. Based on these curves, it seems there is an advantage hydrodynamically to using the more open design of the full scale prototype.

The permeability of each filter element was determined to ascertain its flow performance. Based on the definition of permeability given previously, the higher the permeability the greater the flow capacity through the filter. The permeability of the two different scale filter samples are presented in Table 2. A significant difference in permeability was produced between the two MERV media, almost a two-fold difference, even though there was a moderate $(28 \%)$ difference in rated efficiency. Permeability was not expected to change with gravity levels.

Light sheet imaging was used a diagnostic tool to characterize the upstream flow and associated particle transport. The picture in Fig. 6 clearly shows a particle cloud emanating from the particle generator (left). The image also shows that the particle cloud spreads quickly after leaving the particle generator. While the particle flow structures visible in the particle cloud appear to disperse with downstream distance, the short length of the inlet section did not provide sufficient time for proper homogeneous dispersion of the particle flow at the upstream sampling station. However, it was found that the placement of the upstream sampling probe slightly away from the facility center line provided consistent readings of particle counts with the downstream probe, in the absence of any filter elements. This configuration was used for filter testing. 


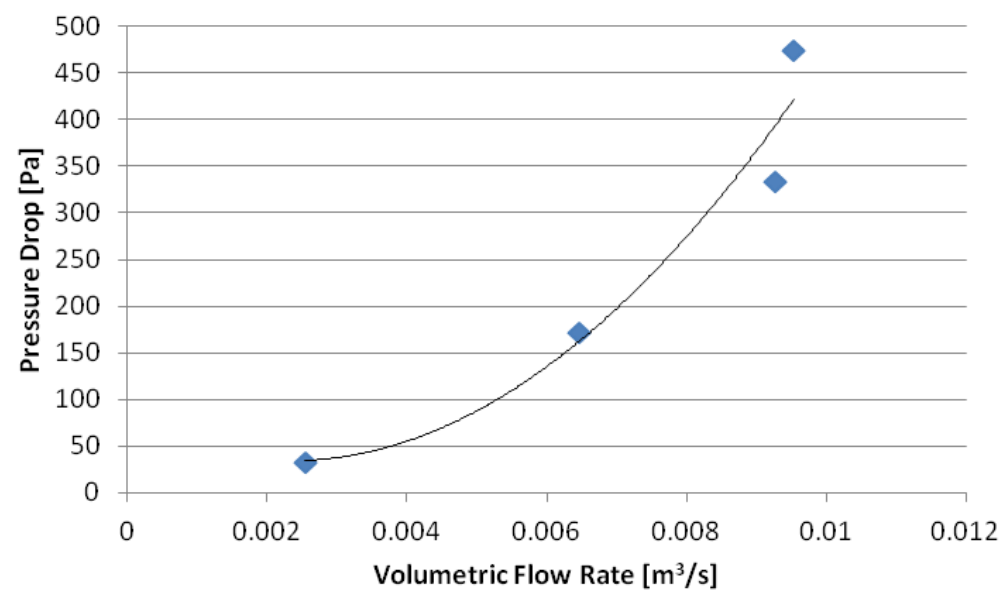

(a)

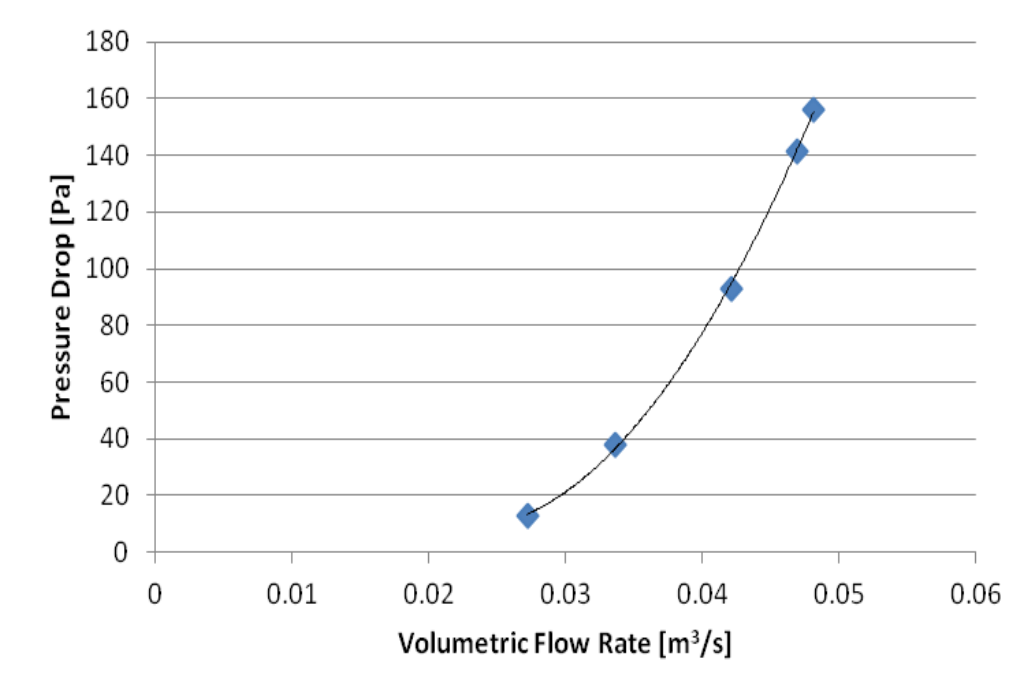

(b)

Figure 5: Impactor stage performance curves (a) scaled prototype, (b) full scale prototype

Table 2: Filter media permeability

\begin{tabular}{|l|l|}
\hline Filter type & $\begin{array}{l}\text { Permeability @ 100 Pa } \\
{\left[\mathrm{m}^{3} \text { vol. flow/m } / \mathrm{m}^{2} \text { area/s }\right]}\end{array}$ \\
\hline Full Scale Scroll filter (MERV 11) & 0.13 \\
\hline 1/8th scale scroll filter (MERV 13) & 0.07 \\
\hline
\end{tabular}




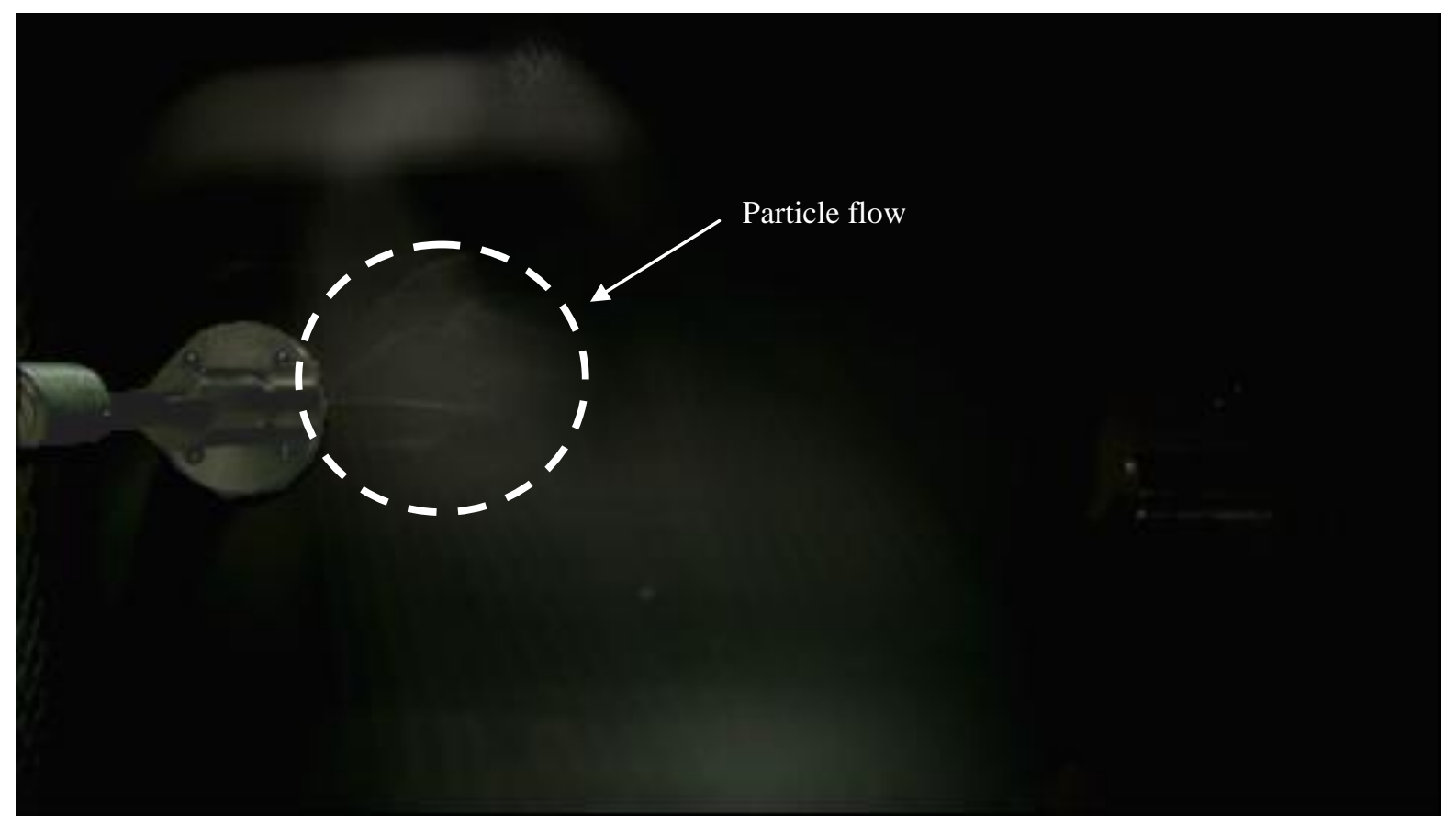

Figure 6: Laser sheet image of upstream particle flow (the particle generator and particle flow are enhanced in the image to aid the reader).

\section{B. Filtration testing}

Particle penetration, or capturing efficiency, performance was obtained for two filter configuration cases: one with only the impactor filter stage, and the second with the combined impactor and scroll filter stages. First a note of caution should be highlighted here. We are using testing methods designed for media filters, in which flow patterns are expected to be steady and remain fairly rectilinear. This may not be the case here because of the use of the impactor stage which produces local flow curvature and the inclusion of a wiper device that can cause an additional level of flow complexity. The effects of flow unsteadiness can be factored out through statistical sampling over sufficiently long times, while to account for the flow divergence, or curvilinear motion, one must assume that there are direct streampaths between the upstream and downstream sampling probe. The collection efficiency is presented here with these caveats. In addition, the laboratory data was considered statistically more significant than the flight data because of the greater number of tests that were performed and longer sampling times obtained. Another aspect of the low-g measurement is that operations during the flight were challenging because of variations in low-g levels and the transition from high-g to low-g levels that affected the transport of the solid particles as they left the particle generator. Lastly, the shorter distance between the particle generation source and the upstream particle sampling probe in the scale prototype further limited particle dispersion, which most likely produced more conservative upstream particle count numbers and thereby skewed the capturing efficiency calculations. Therefore, the results presented in this section are focused mostly on the collection efficiency data of the full scale prototype, with some comparison to the data from the scaled prototype.

Figure 7 gives the efficiency data for the impactor stage alone. The tests were nominally conducted at face velocity of $0.5 \mathrm{~m} / \mathrm{s}$ to simulate the nominal design flow rates. However, one of the tests was performed at $0.4 \mathrm{~m} / \mathrm{s}$ to match the maximum velocity attainable in the impactor and scroll stage configuration (described next). The error bars in the plots represent the relative error for each size bin. The graph shows that the laboratory data sets, at the two different flow speeds, tend to follow each other closely over most of the particle range. Also, there seems to be an indication that the presence of the wiper had little to no effect on the filtration performance as can be seen by comparing the two plots at $0.5 \mathrm{~m} / \mathrm{s}$. In contrast, the low-g data is significantly different at the smallest particle sizes up to $2.5 \mu \mathrm{m}$, while at the largest particle sizes it is closer to the laboratory data. In general, no indication of a classical impactor cut size curve, as described in section II.A, could be discerned, particularly with the laboratory data. While the flight data does show some signs of a cut curve, the level of particle capturing remained significantly 
high below the particle cut size. There are a few possibilities for the high efficiencies below the cut size in the laboratory and flight data. For one, there could be excessive particle settling in the laboratory tests due to the use of the dense simulant particles used, that would result in a miss-representation of higher particle collection on the impactor stage below the cut size. However, because capturing efficiencies are expected to approach $100 \%$ above the cut size, i.e. low to no downstream particle counts, the enhanced particle settling did not affect the data above the cut size. Another effect can be connected to the presence the collection belt which provides two consecutive bluff surfaces in the flow after the impactor orifice. The wake flow from the first surface could interact with the second surface providing additional particle capturing on this surface. If so there would be an added benefit to the belt configuration. Further studies into this aspect of the impactor stage, or the nature of the low counts on the downstream probe, will be worth pursuing.

The capturing efficiency for the two combined stages, impactor and scroll filter, is given in Fig. 8. Note first that because of the higher flow resistance of the stacked impactor and scroll stage configuration and the power limitation on the vacuum cleaner blower, the highest face velocity attainable in the full scale prototype was $0.4 \mathrm{~m} / \mathrm{s}$. Higher velocities were possible with the scaled prototype. The plots show that there is generally better agreement between the laboratory and low-g data in the range of larger particle sizes, down to the $2.5 \mu \mathrm{m}$, with achieved efficiencies greater than $99 \%$. The capturing efficiencies remained above $99 \%$ in the laboratory data, but the flight data exhibited a gradual drop in efficiency approaching the smallest particle sizes down to a value of $87 \%$ efficiency at $0.3 \mu \mathrm{m}$. The slightly lower efficiencies in the flight tests are most likely due to edge leaks resulting from the slack in the medium. This is because the filter system lacked a media tensioning mechanism, and the slack in the filter medium caused the medium to bow out, due to flow pressure, between the spindles used to form the pleats. This effect also produced gaps between the edge of the medium and the inside duct walls. The filter system was tested under these conditions in the flight tests, while manual tensioning was applied on laboratory tests. The flight data also had much larger relative error as indicated by the error bars in the plot that may be attributed to the limited data sets and possibly from transients arising from the g-level transitions into the low-g periods of the flight. The error bars for the laboratory data are too small to be seen in the plots, and the error bar for the scale prototype flight could not determined because of the more restricted number of test runs The efficiencies presented in this graph are the aggregate efficiencies of the impactor stage and scroll filter stage. Therefore, a comparison of the plots in Fig. 7 and 8 shows that, in the laboratory tests, the scroll filter stage added another $4 \%$ in collection efficiency. However, it needs to be determined whether the high efficiencies attributed to the impactor stage are a real effect of enhanced collection or due to some other undetermined effect.

After the filter medium and impactor collection surfaces were loaded with an accumulation of particles from multiple test runs, the self-cleaning operation was performed. The clean side of the collection belts on the impactor stage were translated half way around the belt loop to face upstream, while the filter medium on the scroll stage was rolled up on the collection spool in order to expose the flow to a fresh portion of the medium. Pictures of the loaded impactor stage belts are shown in Fig. 9. The particle deposits show up as streaks on the full scale prototype because of the use of orifice slots, while distinct circular spot deposits are visible in the loaded impactor bands of the scale prototype produced by the arrays of millimeter diameter orifice holes. The indexing (or scrolling) operation on the scroll stage was activated by switching on the servo motor. This operation was observed during several trials which consistently showed that the loaded filter medium rolled up tightly and orderly on the collection spool. The power draw on the servo motor was 7.2 watts at a rotation rate of 0.26 revolutions per second. Fig. 8 shows a comparison of efficiencies before and after the scrolling of the filter medium. The two plots generally agree well. For these two cases, the medium was manually tensioned using a hand crank at the supply spool to mitigate the medium bowing effect. The similar high levels of efficiency before and after scrolling of the medium show that there was no adverse effect produced by the self-cleaning operation of scrolling the medium. 


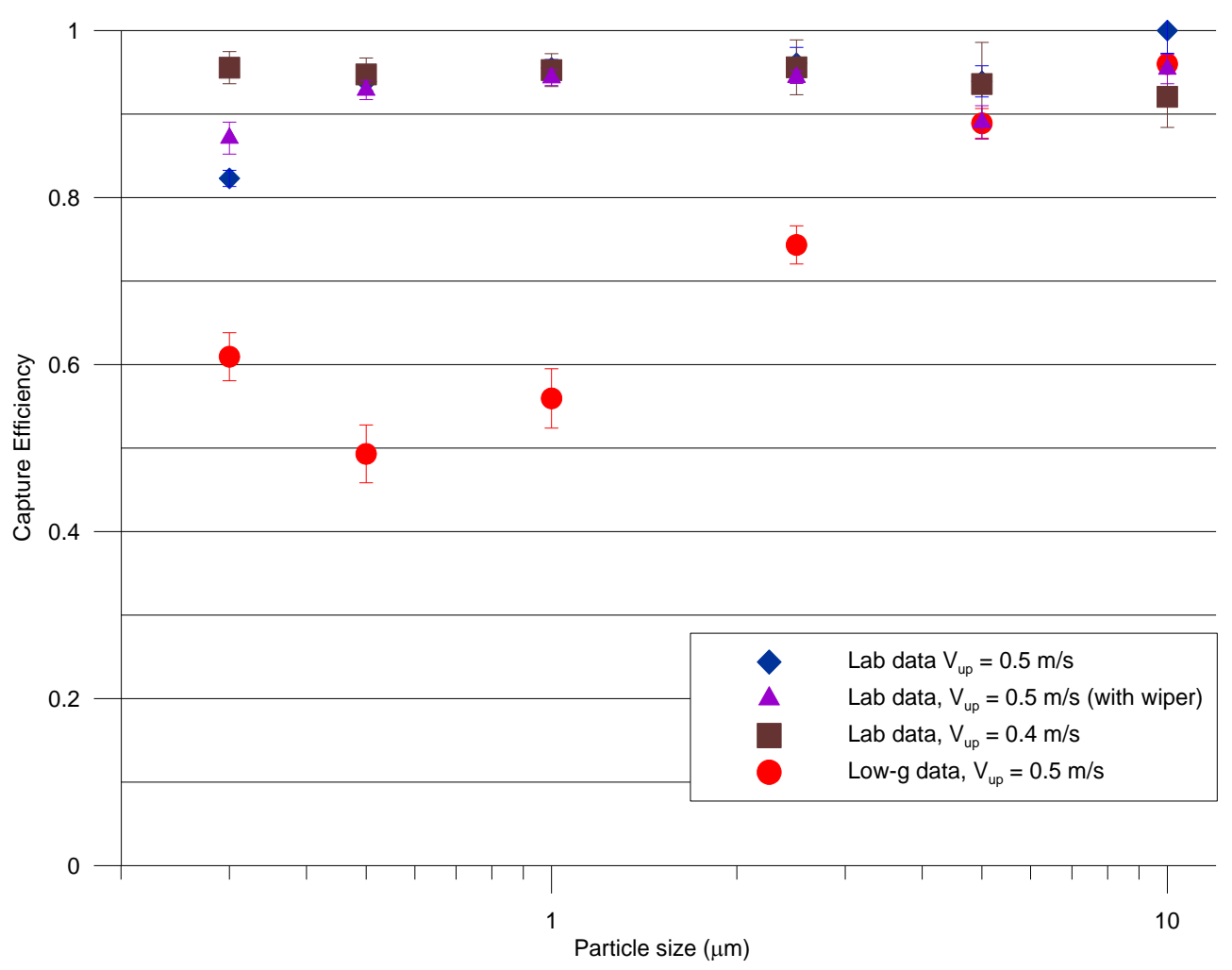

Figure 7: Particle Collection Efficiency of Impactor Stage (error bars represent relative error).

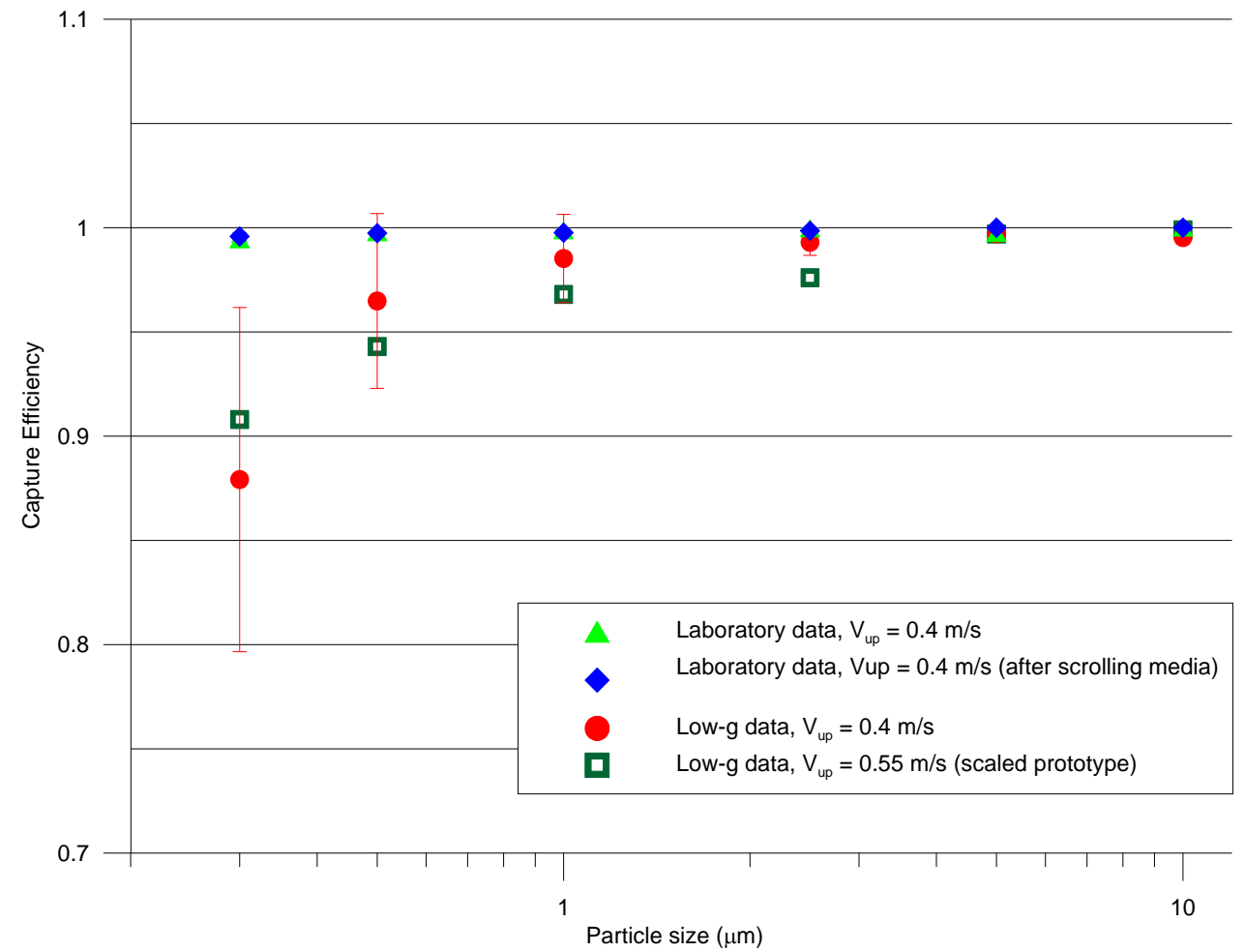

Figure 8: Particle collection efficiency of combined filter system (error bars are the relative error). 


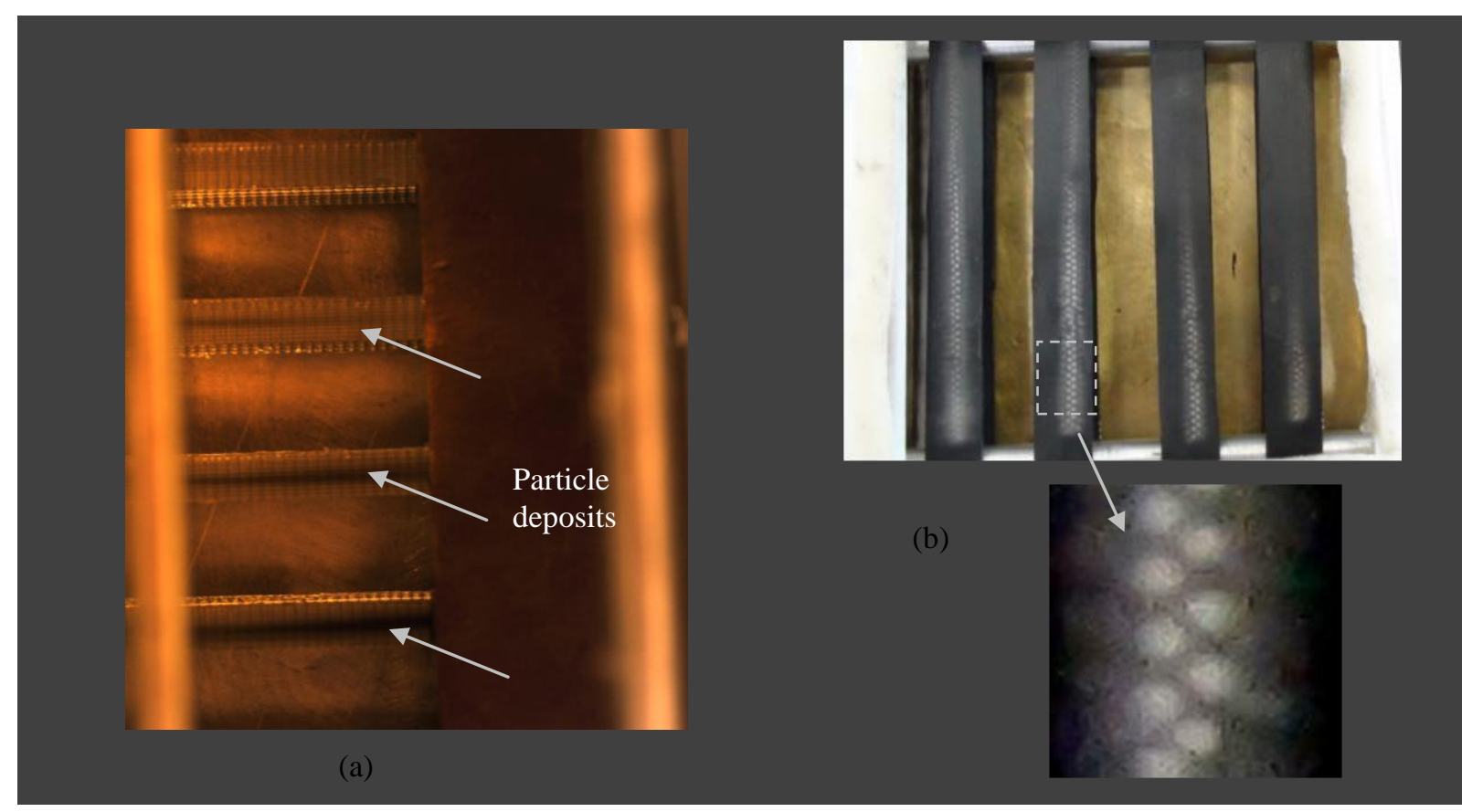

Figure 9: Picture of impactor collection bands after regeneration. (a) Full scale prototype, (b) scale prototype (the loaded belt surfaces were translated to other side of the impactor stage for viewing purposes).

\section{Conclusion}

A novel air filtration system for crewed vehicles and extraterrestrial outposts has been developed through a partnership between NASA and Aerfil. The filter system consists of several stages of filtration including an inertial impaction stage, an indexing (or scroll) media stage, and a final high efficiency filter media stage. An 1/8-th scale and full scale prototype of the filter system were constructed and subsequently tested at NASA GRC under laboratory ambient conditions and on the Zero-G Corp aircraft in low gravity. The tests demonstrated the filter system provided good overall performance. Particle collection efficiencies of $99 \%$ and greater were found for the system as a whole in the laboratory tests, and slightly lower in the flight tests. The scroll filter media self-cleaning operation was also shown to perform satisfactorily at relatively low power. There was general agreement between the ground and laboratory tests, but discrepancies were found at the smallest particle sizes. Additional testing at low$\mathrm{g}$ conditions may be required to resolve this discrepancy. Areas of improvement were identified through testing of the filter system. These included better sealing on the edges of the filter medium, relocation of wiper device, and provision for tensioning the scroll filter medium.

\section{Appendix A: Glossary of keywords}

$d_{50}:$ The particle diameter at which $50 \%$ of particles are collected on the impaction plate and the other $50 \%$ penetrate through the impactor.

Depth Filtration: The trapping of particles as they pass through the tortuous path between fibers in fibrous filters.

Filter efficiency: A measure of the particle collection performance of the filter element obtained through a ratio of the number of particles trapped on the filter and the number of particles found in the air or gas upstream of the filter.

American Institute of Aeronautics and Astronautics 
Permeability: A measure of a medium's capacity for the filtration typically calculated from the measured flow rate at a defined pressure drop.

Penetration: The ratio of particles counts found downstream of the filter to particle counts upstream of the filter.

Relaxation time: A characteristic time that is required for a particle in the flow to adjust (or relax) to new flow conditions.

\title{
Appendix B: Acronyms
}

\author{
HEPA High Efficiency Particulate Air \\ IPP Innovative Partnership Program \\ ISRU In-Situ Resource Utilization \\ MERV Minimum efficiency reporting value
}

\section{Acknowledgements}

We will like to acknowledge several individuals for their valuable support and assistance in this development. We thank Mr. Vinay Bothra of Spectrum Filtration for his company's fabrication support of the full scale prototype; Dr. Marcos Aldoph of Trox do Brasil for providing the HEPA filters used in testing; Dr. Jeff Mackey of Vantage Partners LLC for his role in setting up the flight rig; Mr. Mark Hyatt of NASA GRC for his very supportive project manager role; and Larry Ost of Filtration Group Incorporated for his role in the fabrication of the scale prototype. We also thank all those involved in the various flight campaigns providing ground support and participating in flight operations: Mr. Vinay Bothra, Dr. Marcos Aldoph, Dr. Vinod Vijayakumar (Aerfil), Dr. Jeffrey Mackey, Mr. Mark Hyatt, and Mr. Logan Larson (University of Michigan).

\section{References}

${ }^{1}$ Hinds, W.C. Aerosol Technology: Properties, Behavior, and Measurement of Airborne Particulates, $2^{\text {nd }}$ Ed., Wiley-Interscience, New York, 1999, Ch. 5.

2 "In Situ Solid Particle Generator." NASA Tech Briefs, Jan. 2013 issue (vol. 37, no. 1)

3 American Society of Heating, Refrigeration and Air-Conditioning Engineers (2007) "Method of Testing General Ventilation Air-Cleaning Devices for Removal Efficiency by Particle Size," ASHRAE 52.2-2007

${ }^{4}$ Institute of Environmental Sciences and Technology (2007) "HEPA and ULPA Filters,", IEST-RP-CC001.5

${ }^{5}$ International Organization for Standardization (2011) "High-efficiency filters and filter media for removing particles in air -- Part 2: Aerosol production, measuring equipment and particle-counting statistics," ISO 29463-2.

${ }^{6}$ Orbitec Corp. (2006) "CHARACTERIZATION SUMMARY OF JSC-1AF LUNAR MARE REGOLITH SIMULANT,” URL: http://www.orbitec.com/store/JSC-1AF_Characterization.pdf. 